\title{
PIVET rFSH dosing algorithms for individualized controlled ovarian stimulation enables optimized pregnancy productivity rates and avoidance of ovarian hyperstimulation syndrome
}

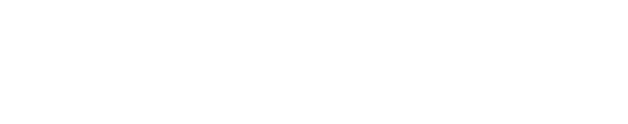

John L Yovich ${ }^{1,2, *}$

Birgit Alsbjerg ${ }^{3,4, *}$

Jason L Conceicao'

Peter M Hinchliffe'

Kevin N Keane ${ }^{1,2, *}$

'PIVET Medical Centre, Perth, ${ }^{2}$ School of Biomedical Science, Curtin Health Innovation Research Institute Bioscience, Curtin University, Perth, WA, Australia; ${ }^{3}$ The Fertility Clinic, Skive Regional Hospital, Skive, ${ }^{4}$ Faculty of Health, Aarhus University, Aarhus, Denmark

*These authors contributed equally to this work
Correspondence: John L Yovich PIVET Medical Centre, 166-168 Cambridge Street, Leedervile, Perth, WA 6007, Australia Email jlyovich@pivet.com

\begin{abstract}
The first PIVET algorithm for individualized recombinant follicle stimulating hormone (rFSH) dosing in in vitro fertilization, reported in 2012, was based on age and antral follicle count grading with adjustments for anti-Müllerian hormone level, body mass index, day-2 FSH, and smoking history. In 2007, it was enabled by the introduction of a metered $\mathrm{rFSH}$ pen allowing small dosage increments of $\sim 8.3$ IU per click. In 2011, a second rFSH pen was introduced allowing more precise dosages of 12.5 IU per click, and both pens with their individual algorithms have been applied continuously at our clinic. The objective of this observational study was to validate the PIVET algorithms pertaining to the two $\mathrm{rFSH}$ pens with the aim of collecting $\leq 15$ oocytes and minimizing the risk of ovarian hyperstimulation syndrome. The data set included 2,822 in vitro fertilization stimulations over a 6-year period until April 2014 applying either of the two individualized dosing algorithms and corresponding pens. The main outcome measures were mean oocytes retrieved and resultant embryos designated for transfer or cryopreservation permitted calculation of oocyte and embryo utilization rates. Ensuing pregnancies were tracked until live births, and live birth productivity rates embracing fresh and frozen transfers were calculated. Overall, the results showed that mean oocyte numbers were 10.0 for all women $<40$ years with $24 \%$ requiring $\mathrm{rFSH}$ dosages $<150$ IU. Applying both specific algorithms in our clinic meant that the starting dose was not altered for $79.1 \%$ of patients and for $30.1 \%$ of those receiving the very lowest rFSH dosages ( $\leq 75 \mathrm{IU}$ ). Only $0.3 \%$ patients were diagnosed with severe ovarian hyperstimulation syndrome, all deemed avoidable due to definable breaches from the protocols. The live birth productivity rates exceeded $50 \%$ for women $<35$ years and was $33.2 \%$ for the group aged 35-39 years. Routine use of both algorithms led to only $11.6 \%$ of women generating $>15$ oocytes, significantly lower than recently published data applying conventional dosages $(38.2 \% ; P<0.0001)$. When comparing both specific algorithms to each other, the outcomes were mainly comparable for pregnancy, live birth, and miscarriage rate. However, there were significant differences in relation to number of oocytes retrieved, but the mean for both the algorithms remained well below 15 oocytes. Consequently, application of both these algorithms in our in vitro fertilization clinic allows the use of both the rFSH products, with very similar results, and they can be considered validated on the basis of effectiveness and safety, clearly avoiding ovarian hyperstimulation syndrome.
\end{abstract}

Keywords: IVF, OHSS, AFC, AMH, BMI

\section{Introduction}

The first live births from in vitro fertilization (IVF) were achieved from single oocytes recovered from monitoring natural cycles and detecting the luteinizing hormone surge. 
However, higher rates of pregnancy and live births were found after ovarian stimulation followed by human chorionic gonadotrophin (HCG) trigger to recover several oocytes in a controlled fashion. Currently, ovarian stimulation is most often undertaken with recombinant follicle stimulating hormone $(\mathrm{rFSH})$, and the starting dose of this gonadotrophin is important for achieving optimal numbers of oocytes in order to produce viable embryos. However, the response needs to be tempered to diminish the risk of ovarian hyperstimulation syndrome (OHSS), a potentially lethal condition, occurring at increased likelihood when oocyte numbers exceed 15. At present, most clinicians still rely on a standard dose of rFSH, typically 150-225 IU, with adjustments based on each clinician's experience and the patient's previous response. The earliest attempts to develop algorithms based on various biological and anthropometric parameters ensued from an academic IVF group in Copenhagen ${ }^{1,2}$ who constructed their risk algorithm based on antral follicle count (AFC), ovarian volume, Doppler score, age, and smoking habits. However, some of these parameters are not commonly used, measured, or not obtainable in most clinics. Howles et $\mathrm{al}^{3}$ evaluated several potential variables and identified four critically important parameters: FSH level at screening, body mass index (BMI), age, and number of follicles $<11 \mathrm{~mm}$ and found them to be the most predictive factors. A formula was calculated using the CONSORT algorithm based on these four variables. This algorithm was tested in a multicentre study on 172 normoovulatory women aged 18-34 years, and a mean of 10.3 oocytes was retrieved. However, the cancellation rate was as much as $17 \%$, which was rather high in this potentially good-responder group. ${ }^{4}$

Recently, PIVET Medical Centre published an algorithm ${ }^{5}$ designed to calculate the appropriate dosage of $\mathrm{rFSH}$ to provide 8-12 oocytes from all women within the IVF program and to reduce the risk of severe OHSS. The algorithm covered all patients from very poor responders to hyperresponders. The rationale behind that algorithm was to target those women at higher risk for OHSS with smaller dosages of rFSH than was standard practice. This was enabled by the introduction of the Puregon Pen by Merck Sharp \& Dohme (Sydney, NSW, Australia) (MSD) into Australia in 2007, which enabled a dial-up dosage of 25 IU increments, but with three subdivisional clicks assumed to be approximately 8.3 IU each (Figure 1). This algorithm then allowed much lower dosages to be provided to women with high AFC ratings and higher anti-Müllerian hormone (AMH) levels, and the dose was adjusted downwards for younger women and those with low BMI ratings.
The original PIVET algorithm enabled rFSH dosages for the Puregon Pen, ${ }^{5}$ which were much lower than the usually administered 150-225 IU, yet still permitted an adequate response but with minimal risk of over-response and the associated concomitant risk of severe OHSS. According to the algorithm, such dosage could range as low as 41.7 IU daily for very young women with BMI as low as $16 \mathrm{~kg} / \mathrm{m}^{2}$, often with typically enlarged polycystic ovaries and AMH levels $>30 \mathrm{pmol} / \mathrm{L}$. Such women had "targeted mild stimulation" but still had the benefits of sufficient oocytes to enable blastocyst culture and single embryo transfers (ETs) as well as the benefit of some embryos for cryopreservation facilitating a higher pregnancy productivity rate ${ }^{6}$ from a single transvaginal oocyte aspiration (TVOA). From our perspective, this strategy is superior to the idea of minimal stimulation protocols using clomiphene citrate, where few eggs are recovered, many cases are cancelled, and minimal cases have the benefit of additional cryopreserved embryos for future use. ${ }^{7}$

At the time of the algorithm study (October 2009), only the Puregon Pen enabled small dosage increments ( 8.3 IU), while the competitive Gonal-f Pen (Merck Serono, Sydney, Australia) allowed only 37.5 IU increments, which in our pilot studies proved to be unsatisfactory in achieving our aims. Subsequently, Merck Serono introduced a new pen early in 2011, which enabled three clicks between the previous dosages, that is, increments of $12.5 \mathrm{IU} /$ click and filled by mass so that rFSH quantity was assured. We elected to design two new algorithms, one adjusted to 12.5 increments for the Gonal-f Pen (Figure 2), and a revised version of the original Puregon algorithm (Figure 1), that captures the current trend in a reduction of maximal $\mathrm{rFSH}$ dosages from 600 to $450 \mathrm{IU}^{5}$, thus providing clinicians with the option to use either of the rFSH products with a view to targeted low-dose stimulation of those women at increased risk of OHSS.

The current observational study analysed IVF data following stimulation using either PIVET algorithms, and adjusted for 8.3 IU or 12.5 IU increments over a continuous 6-year period. The clinical aims were to collect eight to twelve oocytes for optimal benefit and four to eight oocytes in the very-high-risk group, while maintaining a minimum risk of overstimulation. These data showed that routine application of the PIVET algorithms respective for each drug makes these aims eminently feasible.

\section{Materials and methods Study design}

This retrospective study covers a continuous 6-year period at PIVET (April 2008 to April 2014) during which period both 


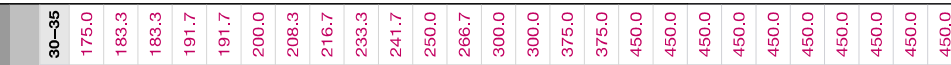

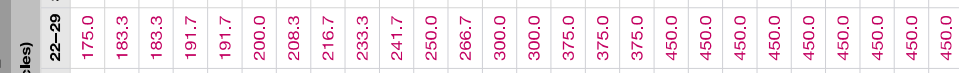

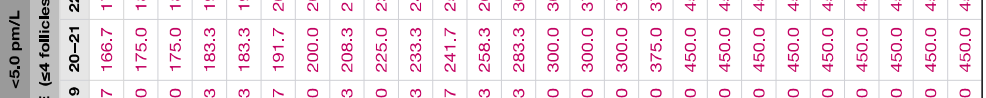

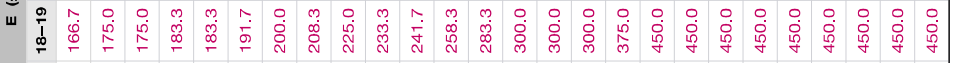

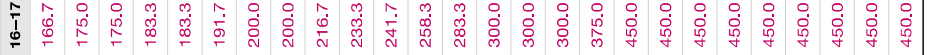

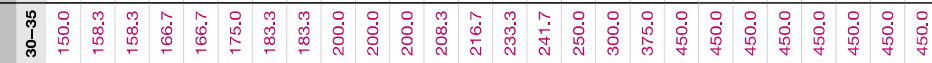

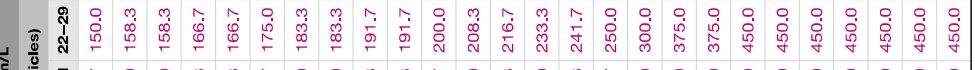

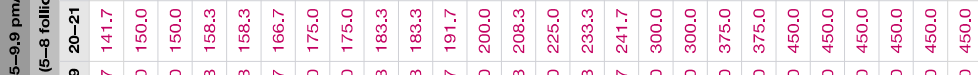

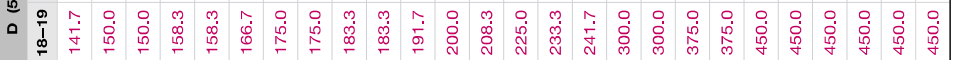

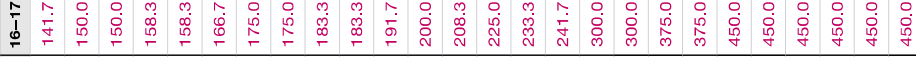

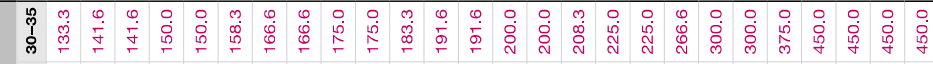

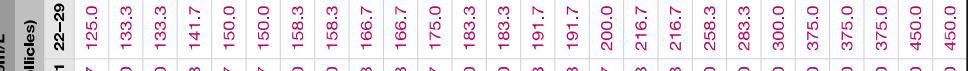

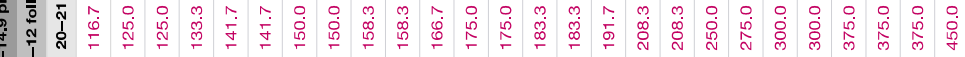

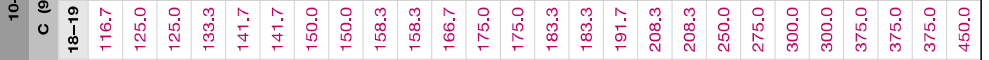

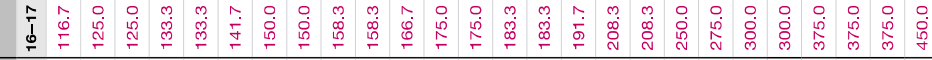

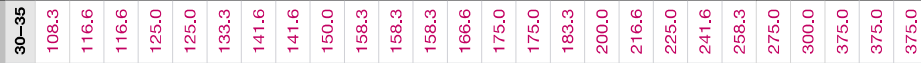

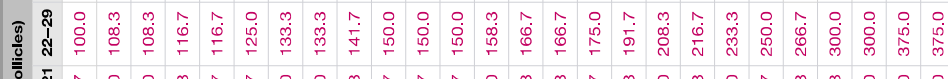
$\begin{array}{lll} & \\ 0\end{array}$ 至

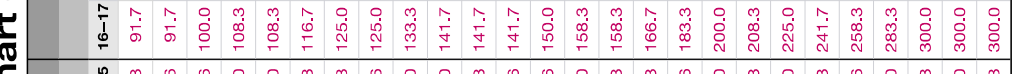

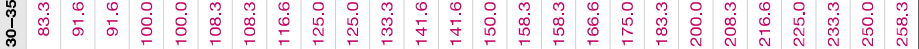

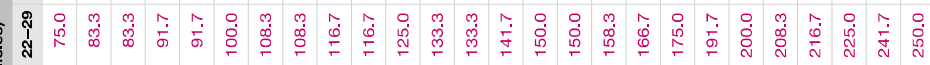

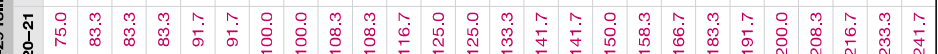

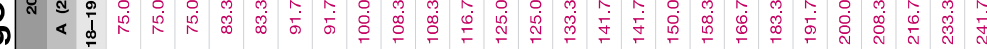

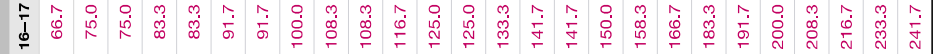

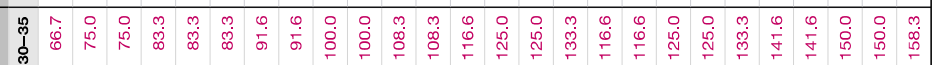

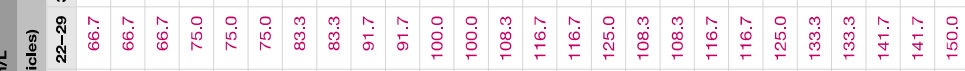

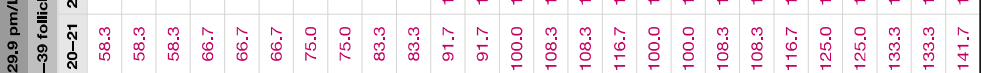

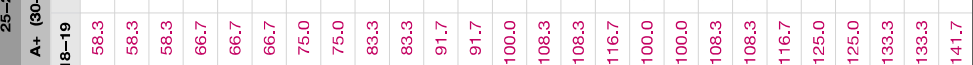

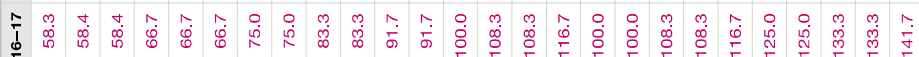

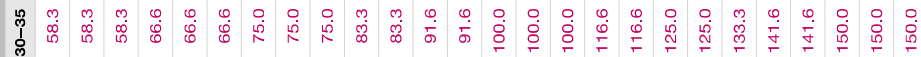

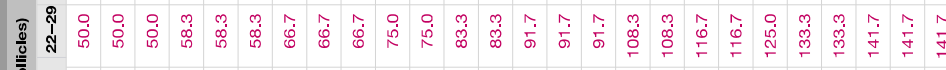

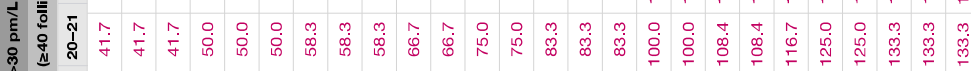

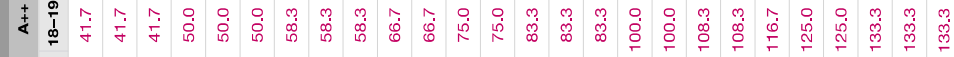

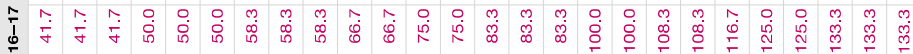

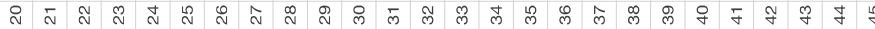
章谤言
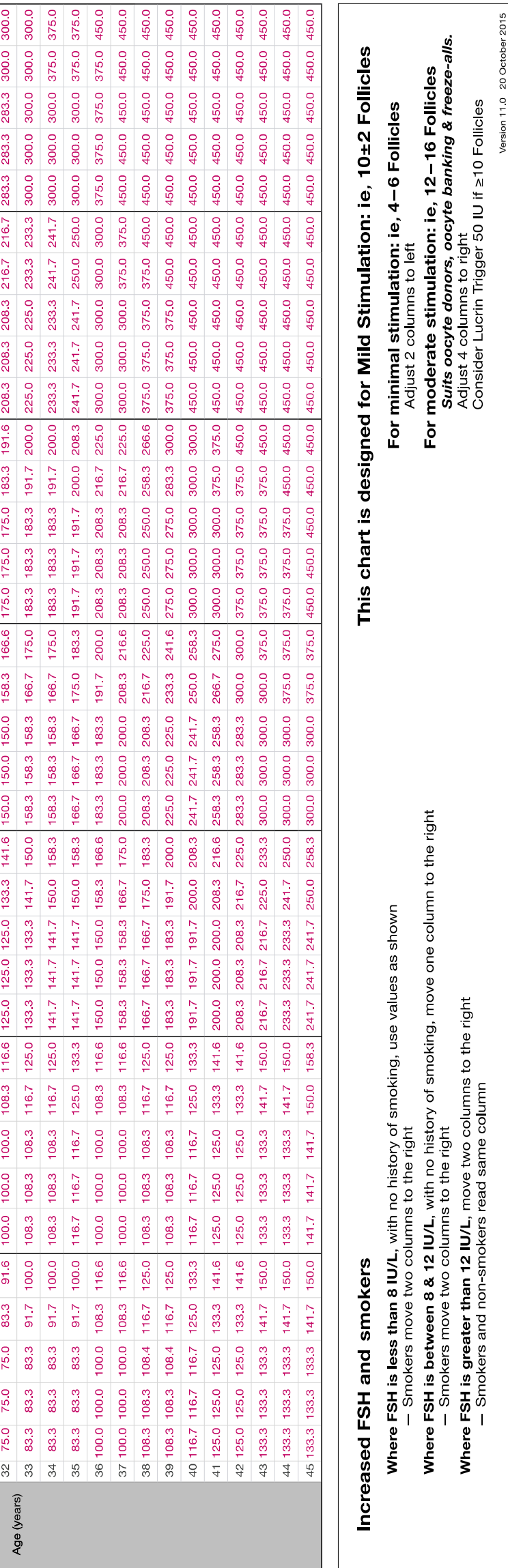

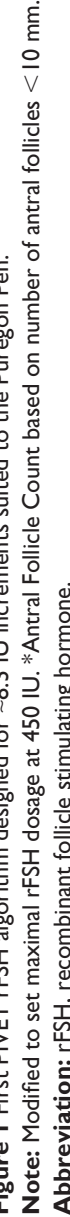




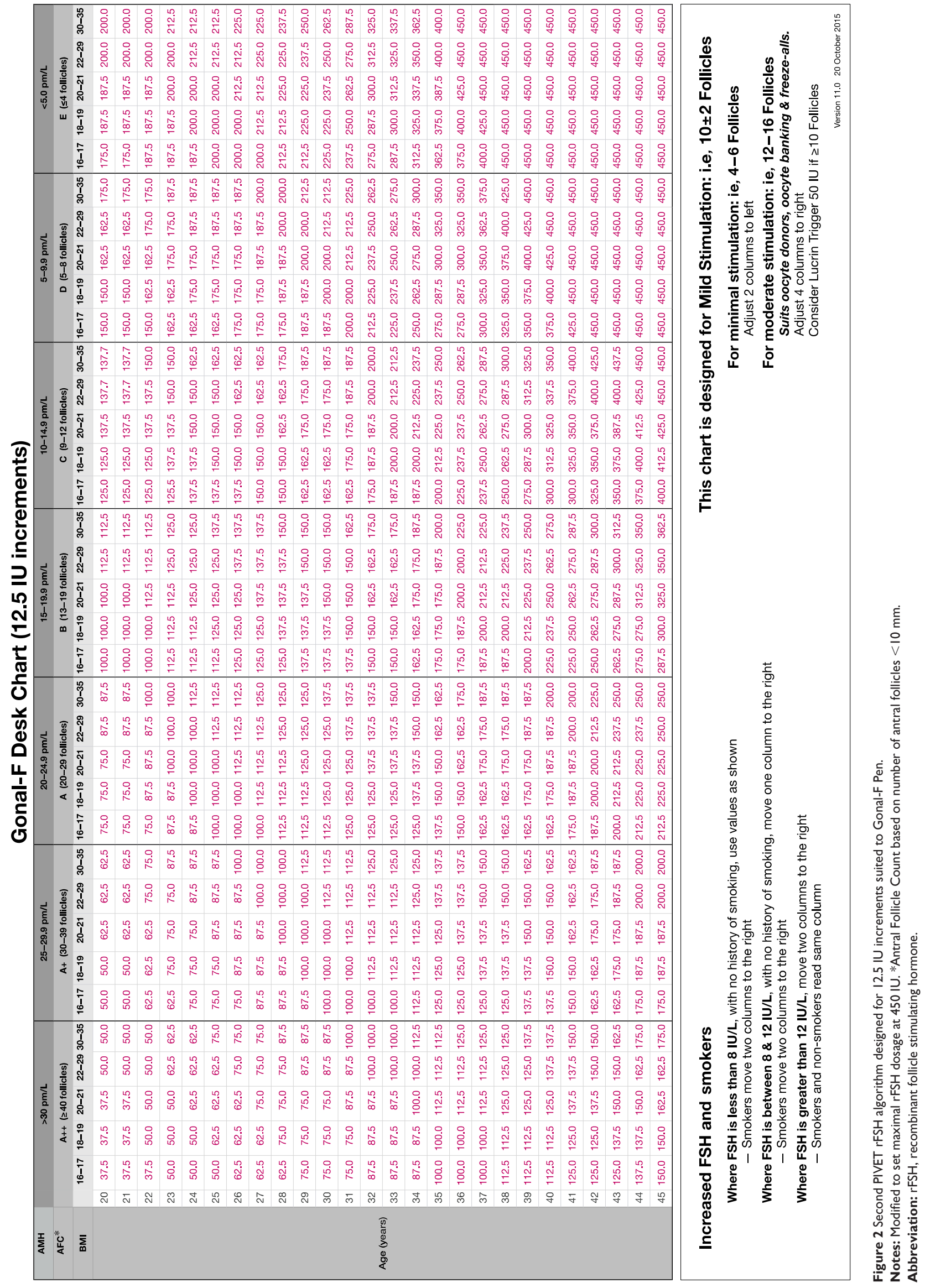


the algorithms were used to determine the rFSH starting dose. The data were drawn from our entire database containing all cases treated in this 6-year period (Figure 3). The data period enabled all pregnancies to be tracked through to deliveries. Few patients with $\mathrm{BMI}>36 \mathrm{~kg} / \mathrm{m}^{2}$ were treated during the entire period, and they were treated according to the algorithms as if the BMI was $35 \mathrm{~kg} / \mathrm{m}^{2}$. The protocol was the same as applied in our previous publication, ${ }^{5}$ but the new algorithm for Gonal-f was introduced in April 2011 with the launch of the new pen. Dosages were adjusted to be close to the original Puregon dosages, and both the rFSH products were used routinely based on clinicians' choice and availability in the current study. All clinical and laboratory management of IVF or Intracytoplasmic Sperm Injection (ICSI) procedures was not altered during the entire 6-year study period, apart from trends in ETs, namely more cases committed to blastocyst culture, more single ETs, and more cases with selective cryopreservation of the better quality embryos.

\section{Patient management}

All IVF patients completed informed and written consent when undergoing an assessment cycle, which occurred following their primary consultation and within 1-2 months immediately prior to the IVF treatment cycle. This included serum day-2 FSH, AMH, and AFC measured day $5 \pm 1$ along with cycle tracking, a hysterosalpingo-contrast-sonography (HyCoSy) test day 7 to day 9, periovulatory postcoital evaluation, and mid-luteal serum progesterone (P4). At this latter, mid-luteal point, the couple were reviewed for the consideration of treatment proposals. For those proceeding to IVF, the clinician decided the stimulation protocol, and the starting gonadotrophin dosage was calculated from the appropriate algorithm. However, if a further fresh cycle was planned, the essential measurement parameters were repeated in the month prior to the cycle and the rFSH dosage calculated at the day 21 pre-IVF check. Clinicians were instructed to avoid adjusting dosages on the basis of previous responses

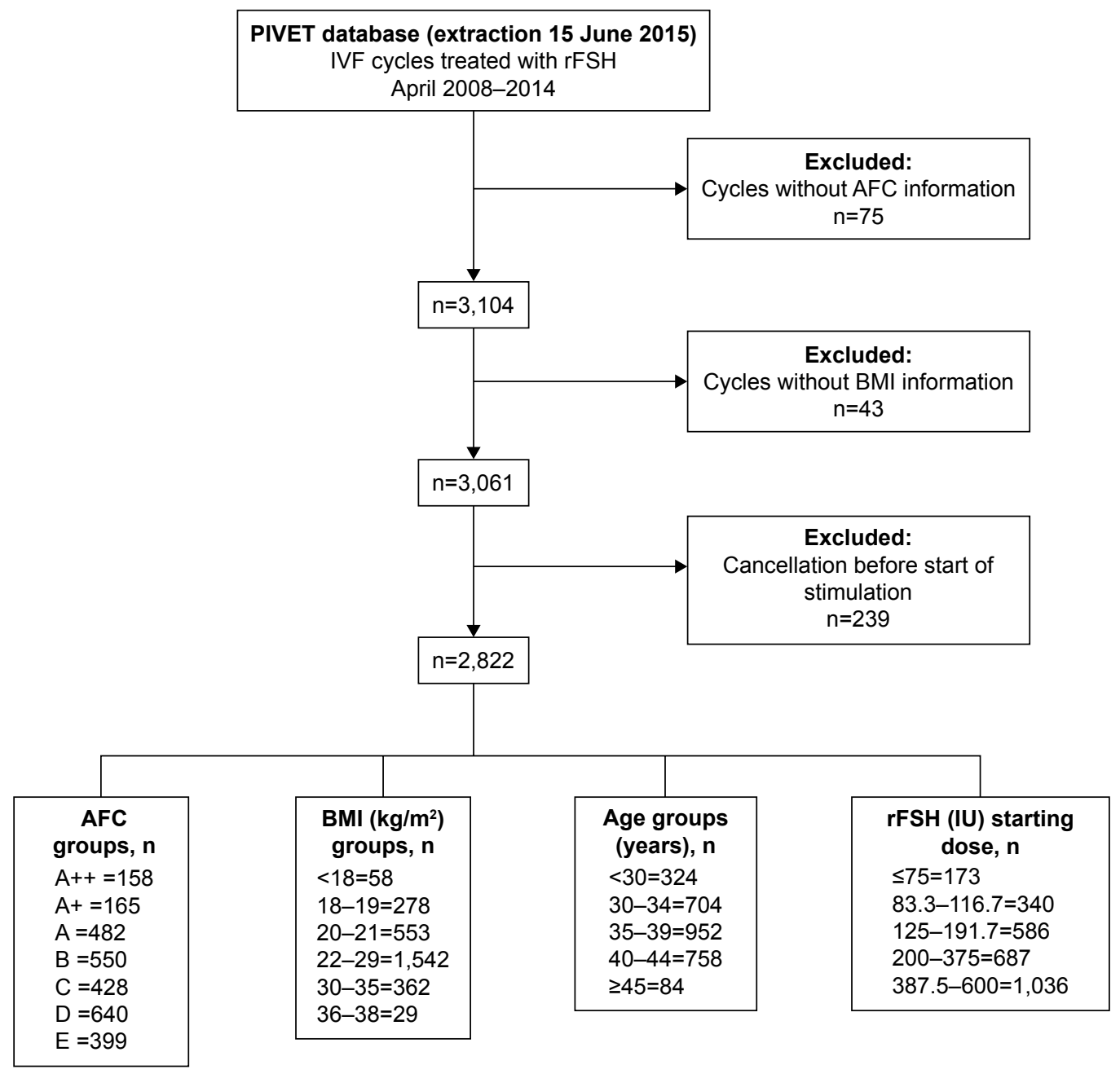

Figure 3 Derivation of 2,822 cases of IVF initiated over a 6-year period at PIVET Medical Centre applying PIVET algorithms. Abbreviations: AFC, antral follicle count; BMI, body mass index; IVF, in vitro fertilization. 
(eg, oocytes collected) but to simply calculate using the updated parameters.

\section{PIVET algorithm development}

Examination of more than 5,000 IVF cycles along with tracking the destiny of more than 50,000 recovered oocytes created a profile of those protocols leading to the development of eight to twelve follicles and oocytes according to the background AFC ratings. These data were then compared with the risk charts (chance of pregnancy vs risk of OHSS) published from the Copenhagen Group. ${ }^{1,2}$ As the algorithms evolved, we applied data modeling and graphic smoothing techniques to create a continuously rising dosage schedule, without irregularity. As previously stated, we commenced by applying the Puregon Pen, which is filled by bioassay and provides MSD-guaranteed dosages of $25 \mathrm{IU}$ rFSH. We made an assumption that each click of the pen provides approximately one-third dosage, that is, $\sim 8.3 \mathrm{IU} / \mathrm{click}$, although this has not been endorsed or confirmed by MSD, hence $\sim 8.3$ IU increments (Figure 1). ${ }^{5}$ Following the release of the Gonal-f Pen (Merck Serono) in 2011, we applied the closest dosage schedule to match the Puregon Pen algorithm, with further data modeling and graphic smoothing techniques to create the model, as shown in Figure 2. Merck Serono indicates that it is this rFSH pen that is filled by mass; hence, all dosage increments of $12.5 \mathrm{IU}$ are fully endorsed and quality assured by Merck Serono.

\section{Selecting rFSH and adjusting starting dosage}

The main parameters relevant to the algorithm are female, age, and current AFC rating, where all detectable follicles $2-10 \mathrm{~mm}$ are included. Definition of ratings are grade $\mathrm{E}, \leq 4$ follicles; grade D, 5-8 follicles; grade C, 9-12 follicles; grade B, 13-19 follicles; and grade A, 20-29 follicles (Figure 3). Patients in the latter grade belong to the highrisk polycystic ovary group and are further classified as grade $\mathrm{A}+(30-39$ follicles) and grade $\mathrm{A}++(\geq 40$ follicles) Figure 3. In selecting the AFC grading, the AMH level was also factored in as a modulator, adjusting the AFC grading category upwards if $\mathrm{AMH}$ implied a higher AFC rating (eg, if the $\mathrm{AFC}$ categorized to $\mathrm{B}$, but $\mathrm{AMH}$ categorized to $\mathrm{A}$, the patients would be categorized as an A grading). Clinicians are advised not to adjust the AFC rating downwards if suggested by a lower AMH reading as this implies a higher $\mathrm{rFSH}$ dosage, often proving inappropriate with excessive ovarian responses. Internal correlation studies at PIVET show high correlation between $\mathrm{AMH}$ and AFC, but there can be discordance in $\sim 11 \%$ of cases (Keane et al; unpublished data, 2016). Moving the AFC rating upwards ensured that a lower dosage of rFSH would be selected, ensuring safety and reducing risk of overstimulation and OHSS. The current $\mathrm{AMH}$ levels were determined using Gen II (Beckman Coulter, Brea, CA, USA). ${ }^{8}$ Other adjustments within the algorithm include BMI, smoking history, and day-2 serum $\mathrm{FSH}$, the latter adjusting 1 or 2 readings to the right (ie, higher rFSH dosage; Figure 1). Patients classified as egg donors or those having egg storage (freeze all) have their rFSH dosage adjusted four readings to the right (ie, higher rFSH dosage), as they will have an antagonist stimulation cycle with agonist (eg, Lucrin, leuprolide acetate; Abbott Australasia Pharmaceuticals, Sydney, NSW, Australia) trigger $^{9}$ and no luteal support drugs because there is no transfer and the risk of OHSS is considered to be low. These data have been included to determine the impact of the algorithms on oocyte retrieval and utilization but excluded for calculations of pregnancy, live births, and miscarriage rates.

\section{Ovarian stimulation regimens}

Women with high AFC ratings (all A categories and some $\mathrm{B}$ if $\mathrm{AFC}>15$ antral follicles) were treated by an antagonist regimen preferentially. Those with low categories (D and E) were treated by a flare regimen with some very poor responders treated by an agonist antagonist conversion with estrogen priming (AACEP) regimen. ${ }^{10}$ Women with AFC category $\mathrm{B}<15$ antral follicles and category $\mathrm{C}$ could be treated by either regimen or even a long downregulation protocol at clinician's discretion. The long down-regulation protocol was also preferred for cases of underlying adenomyosis and endometriosis. This approach accords with the Nelson algorithm. $^{11}$

\section{Adjusting rFSH dosage}

IVF cycle management commences with day-2 parameters of estradiol (E2) $<200 \mathrm{pmol} / \mathrm{L}, \mathrm{P} 4<5.0 \mathrm{nmol} / \mathrm{L}$, and luteinizing hormone $<10 \mathrm{IU} / \mathrm{L}$. FSH levels are measured and may lead to adjustment of the algorithm dosage if different from previous, for example, FSH 20 IU may cause rFSH dosage selection 2 squares to the right (8.3/12.5 to $16.7 / 25$ IU where $<300 \mathrm{IU}$; or 25-37.5 IU where $\geq 300 \mathrm{IU})$. Most cycles utilize the antagonist regimen, with $\mathrm{rFSH}$ commencing on day 3 , but "agonist flare cycles" means that patients commence Lucrin 4 IU on day 2, followed by rFSH day 3. Serum E2 levels are determined at day 7 , and if $>500 \mathrm{pmol} / \mathrm{L}$, the dosage of rFSH remains unchanged. Conversely, lower levels will require $12.5 \mathrm{IU}$ incremental rises in dosage. The antagonist (Orgalutran: MSD or Cetrotide; Merck Serono) $0.25 \mathrm{mg}$ is introduced with E2 levels $>500 \mathrm{pmol} / \mathrm{L}$. Thereafter, E2, P4, 
and luteinizing hormone levels are measured every 2-3 days along with follicle tracking by transvaginal ultrasound from day 9. Most cases present with two or more leading follicles, $>17$ or $(\geq 18) \mathrm{mm}$ on day 12 of the cycle, the most common day for giving the ovulatory trigger, and TVOA is scheduled for 37 hours posttrigger.

\section{Trigger and luteal support}

Recombinant HCG (rhCG: Ovidrel; Merck Serono) was used as ovulation trigger in doses 6,500-13,000 IU (one or two ampoules). However, in cases considered at high risk of developing OHSS ( $\geq 12$ follicles or $\geq 12,000 \mathrm{pmol} / \mathrm{L}$ E2) and treated with an antagonist regimen, GnRHa (leuprolide acetate, Lucrin 50 IU; Abbott Australasia Pharmaceuticals, Sydney, NSW, Australia) was used as the ovulatory trigger. Luteal support was given as described earlier ${ }^{5}$ and includes rhCG 500-1,000 IU days 4, 7, 10, and 13 after TVOA for those cases with $<12$ follicles and $<12$ oocytes according to our long-standing practices, ${ }^{12,13}$ all other cases received P4 $400 \mathrm{mg}$ thrice daily. Cases with $>12$ oocytes were given Cabergoline $1 \mathrm{mg}$ nocte for 10 days and cases $>20$ oocytes had freeze-all except if the trigger was Lucrin.

\section{Intensive monitoring protocol}

Women were classified as OHSS risk at two occasions firstly, at trigger if E2 was $\geq 12,000 \mathrm{pmol} / \mathrm{L}$ or ultrasound revealed $\geq 12$ follicles that were $\geq 12 \mathrm{~mm}$; and secondly, at TVOA if $\geq 12$ oocytes were recovered. Such patients were instructed to record abdominal girth at the umbilicus each day and report the measurement and wellness (especially noting breathing difficulty, urine flow, and color, as well as any nausea and/or vomiting) daily to the clinic nurse. A blood test was performed every third day and was considered for E2 $>6,000$ $\mathrm{pmol} / \mathrm{L}$ or $\mathrm{P} 4>600 \mathrm{nmol} / \mathrm{L}$; such cases having luteal phase support adjusted to exclude rhCG, if previously prescribed. Women exceeding safe parameters were arranged for abdominal ultrasound to check for OHSS (and need for paracentesis), along with urine sample to detect specific gravity (SG) and need for intravenous hydration if $\mathrm{SG}>1,015$.

\section{ET trends}

In the late algorithm period, there were some changing trends in ET policies. Overall, there was a strong Australian drive to minimize multiple pregnancies; hence, elective single ETs were encouraged. There was also a concern that those cycles characterized by rising $\mathrm{P} 4$ levels prior to trigger would result in disturbances affecting embryo-endometrial synchrony, therefore PIVET policy changes encouraged cryopreservation by vitrification of the best-quality embryos, and fresh transfer of those embryos graded in the lower tier. The trend also included an increased blastocyst culture policy. Consequently, fewer embryos became available for transfer or cryopreservation.

\section{Oocyte retrieval and pregnancy outcomes}

Two utilization measures were calculated to summate the proportion of "usable" embryos transferred or vitrified arising from the total number of two pronucleate embryos generated from a single oocyte retrieval procedure (embryo utilization rate), or the proportion of "usable" oocytes transferred and vitrified arising from the total number of oocytes retrieved from a single oocyte retrieval procedure (oocyte utilization rate). The outcomes of treatment cycles are categorized for cycles initiated and fresh cycles per TVOA or per ET. They are categorized as clinical pregnancy rate (detectable foetal heartbeat at 7 weeks) or live birth rate (at least one live, surviving infant after 28 weeks). The pregnancy and live birth productivity rates include the subsequent frozen embryo transfer outcomes arising from the single TVOA procedure from an individual IVF treatment cycle. PIVET introduced this terminology to avoid confusion with the term "cumulative pregnancy rate," which mostly relates to outcomes from more than one IVF treatment cycle. ${ }^{5,6}$ By using productivity rates, the outcomes of IVF treatments, where top-quality embryos are frozen for various reasons, for example, elevated late follicular P4 levels or risk of OHSS, can still be favorably reflected. ${ }^{14}$

\section{Statistical analysis}

Data were analyzed using Prism 6 (GraphPad Software, Inc., La Jolla, CA, USA) applying one-way or two-way analysis of variance for multiple comparisons of the means within groups, including AFC and age groupings. Ratios were compared using $\chi^{2}$ contingency tables applying Fisher's exact test. $P$-values $<0.05$ were considered statistically significant. Multivariate statistical analysis was conducted by logistic regression using SPSS version 22 (IBM Corporation, Armonk, NY, USA).

\section{Results}

A summary of treatment cycle details along with patient age distribution is shown in Table 1 . The mean age for the total women treated during the 73-month study period was 36.2 years, and ages ranged from 20 to 51 years. The most commonly applied stimulation protocols were the antagonist regimen $(57.4 \%$ of cases) followed by the flare protocol (30.1\% of cases). Only a small group of patients $(12.5 \%)$ 
Table I Patient demographics and treatment flow for women initiating $\mathrm{rFSH}$ stimulation under PIVET algorithms

\begin{tabular}{|c|c|}
\hline \multicolumn{2}{|l|}{ Cycle information (01/04/2008 to $30 / 04 / 2014)$} \\
\hline Cycles initiated, $\mathrm{n}$ & 2,822 \\
\hline TVOA, n & 2,646 \\
\hline Cancellation before oocyte retrieval, $\mathrm{n}(\%)$ & $176(6.2)$ \\
\hline No of embryo transfer, $n$ (\% of retrievals) & $2,211(83.6)$ \\
\hline Single embryo transfer, $\mathrm{n}$ (\% of embryo transfers) & $1,588(71.8)$ \\
\hline Increased monitoring program, $\mathrm{n}$ (\% of retrievals) & $483(18.3)$ \\
\hline Cases admitted to hospital, n (\% of started cycle) & $31(I . I)$ \\
\hline Paracentesis, $\mathrm{n}$ (\% severe OHSS) & $9(0.3)$ \\
\hline Freeze all for OHSS (\% of retrievals) & $55(2.1)$ \\
\hline \multicolumn{2}{|l|}{ Age groups } \\
\hline$<30$ years, $\mathrm{n}(\%)$ & $324(11.5)$ \\
\hline $30-34$ years, $n(\%)$ & $704(24.9)$ \\
\hline 35-39 years, n (\%) & $952(33.7)$ \\
\hline 40-44 years, n (\%) & $758(26.9)$ \\
\hline$\geq 45$ years, $n(\%)$ & $84(3.0)$ \\
\hline Mean age $( \pm S D)$, years & $36.16( \pm 5.27)$ \\
\hline \multicolumn{2}{|l|}{ Stimulation protocols } \\
\hline Antagonist, n (\%) & $1,619(57.4)$ \\
\hline Flare agonist, n (\%) & $850(30.1)$ \\
\hline AACEP, n (\%) & $204(7.2)$ \\
\hline Long agonist, n (\%) & $107(3.8)$ \\
\hline Variants, n (\%) & $42(1.5)$ \\
\hline
\end{tabular}

Abbreviations: AACEP, agonist antagonist conversion with estrogen priming; OHSS, ovarian hyperstimulation syndrome; SD, standard deviation; TVOA, transvaginal oocyte aspiration.

were treated by other protocols including the long agonist downregulation protocol and the variant AACEP regimen. The incidence of women needing admission to hospital for various diagnoses post-TVOA is shown in Table 1. In order to track all patients developing OHSS, 483 risk patients had the intensive monitoring protocol (IMP) applied during the period. Furthermore, 308 patients with $>12$ oocytes were treated with Cabergoline to diminish or prevent OHSS symptoms. Nine cases were categorized as severe OHSS requiring paracentesis or drainage of pleural effusion (one case), which comprised $0.3 \%$ of all cases receiving $\mathrm{rFSH}$ stimulation. Only one case of severe OHSS occurred during the reporting period of the first algorithm publication ${ }^{5}$ and that related to a marked violation of protocol. In this full study period, each of the nine cases had identifiable noncompliance issues in four areas of "protocol violation." They included 1) excessive starting dosage (due to adjusting the $\mathrm{AFC}$ rating the wrong way once the AMH level was factored); 2) incorrect elevation of dosage (due to increasing more than 12.5 IU when hormonal or ultrasound monitoring indicated a need for elevation); 3 ) inappropriate trigger when $\mathrm{E} 2>10,000 \mathrm{pmol} / \mathrm{L}$ or follicle number $\geq 12$ (usually because a flare cycle had prevented use of a GnRHa trigger); and finally, 4) wrong use of HCG during the luteal phase (sometimes a patient from a remote location self-administering Ovidrel prior to receiving approval from the clinic during IMP). Each time the OHSS was exacerbated by superimposed pregnancy (so-called late onset OHSS in all nine cases).

The cancellation rate before TVOA was $6.2 \%$ and included all cancellations after starting $\mathrm{rFSH}$ stimulation (ie, initiated cycle). However, from 173 patients (Figure 3) who received low rFSH ( $\leq 75 \mathrm{IU}$ ), only 15 (8.7\%) patient cycles were cancelled. Most cancellations were a result from inadequate responses in the group of younger patients receiving low $\mathrm{rFSH}$ dosage.

As part of the Australia-wide drive to decrease the twin rate, there was an increasing rise in the transfer of single embryos during the study period, being $78.4 \%$ of all transfers with some variation among the age groups. Younger patients ( $<30$ years) had a mean of 1.1 embryos transferred and the mean increased in the elder age groups to a maximum of 1.5 embryos in the group of $40-44$ years. The overall mean ( \pm standard deviation) number of embryos transferred with the PIVET algorithms was $1.28 \pm 0.45$.

The mean number of oocytes collected through the study period was $8.7( \pm 5.7)$ oocytes and was $10.0( \pm 5.6)$ for those $<40$ years (Table 2 ). Women aged under 30 years had $11 \pm 6.2$ oocytes recovered, while women $40-44$ years had lower but acceptable oocyte numbers (mean of 6), and those with the poorest prognosis with age $>45$ years had a mean of four oocytes recovered. When the oocyte distribution was examined in relation to the AFC categories (Table 3), the highest AFC ratings showed a mean number of 12 oocytes, despite the very low $\mathrm{rFSH}$ starting doses. With respect to BMI groupings shown in Figure 3, the mean number of oocytes recovered was equivalent among different BMI groups, ranging from 7.4 \pm 5.9 in the lowest BMI group $\left(<18 \mathrm{~kg} / \mathrm{m}^{2}\right)$ to $8.6 \pm 5.4$ in a higher BMI group (18-19 $\mathrm{kg} / \mathrm{m}^{2}$ ), but these minimal differences across the other BMI groups were not significant.

Table 2 Oocytes recovered and embryo/oocyte utilization rates for all women having a TVOA procedure categorized by age groups

\begin{tabular}{llllll}
\hline & Age group & & & \\
\cline { 2 - 6 } & $<\mathbf{3 0}$ years & $\mathbf{3 0 - 3 4}$ years & $\mathbf{3 5 - 3 9}$ years & $\mathbf{4 0 - 4 4}$ years & $\geq \mathbf{4 5}$ years \\
\hline Mean oocytes retrieved $( \pm S D)$ & $11.02( \pm 6.2 \mathrm{I})$ & $10.20( \pm 5.93)$ & $9.10( \pm 5.48)$ & $6.18( \pm 4.38)$ & $4.07( \pm 4.11)$ \\
Oocyte utilization rate $(\%)$ & 27.8 & 29.5 & 28.3 & 31.2 & 35.0 \\
Embryo utilization rate $(\%)$ & 50.3 & 50.4 & 49.8 & 60.5 & 64.7 \\
\hline
\end{tabular}

Abbreviations: SD, standard deviation; TVOA, transvaginal oocyte aspiration. 
Table 3 Oocytes recovered for all women having a TVOA procedure categorized according to AFC rating

\begin{tabular}{ll}
\hline AFC group & $\begin{array}{l}\text { Mean oocytes } \\
\text { retrieved }( \pm \text { SD })\end{array}$ \\
\hline A++ $(\geq 40$ follicles $)$ & $12.35( \pm 6.85)$ \\
$A+(30-39$ follicles $)$ & $11.82( \pm 5.85)$ \\
$A(20-29$ follicles $)$ & $12.26( \pm 6.16)$ \\
$B(13-19$ follicles $)$ & $9.98( \pm 4.62)$ \\
C $(9-12$ follicles $)$ & $8.33( \pm 4.4 I)$ \\
D (5-8 follicles $)$ & $5.32( \pm 3.70)$ \\
E ( $<4$ follicles $)$ & $4.90( \pm 4.53)$ \\
\hline
\end{tabular}

Abbreviations: AFC, antral follicle count; SD, standard deviation; TVOA, transvaginal oocyte aspiration.

Table 4 shows the pregnancy outcomes categorized according to different age groups. The data show an expected decreasing pregnancy and live birth rate per ET with increasing age groups, from $43.4 \%$ and $36.4 \%$ in the young group, respectively, decreasing to $11.7 \%$ and $7.5 \%$ in the group of 40-44 years, respectively. Some women treated toward the end of study period will not yet have had spare embryos returned in a FET cycle; however, the live birth productivity rate in the young group $(<30$ years) was as high as $57.1 \%$ per initiated cycle and $60.1 \%$ per TVOA (data not shown).

From Figure 3, it can be seen that 513 women $(18.2 \%$ of total) had starting doses $<125 \mathrm{IU}$, and approximately half of these patients did not require any increase in dosage (Figure 4). Even for those starting at $\leq 75 \mathrm{IU}$, one-third had no change in dosage. The overall percentage of patients continuing at their starting dosage was $79.1 \%$ during the entire study period, which demonstrated the accuracy of the combined application of both the algorithms. Examining the data for dosages outside the conventional range of 150-225 IU rFSH, 667 (23.7\%) received $<150$ IU; while $1,343(47.6 \%)$ received $>300$ IU. This means that less than one-third of women $(29.7 \%)$ received conventional dosages of $\mathrm{rFSH}$ during the study period using the PIVET algorithms, and consequently the vast majority of patients received a "milder" form of stimulation.

It was observed that half of the patients had from 6-10 and $11-15$ oocytes retrieved ( $32 \%$ and $22 \%$, respectively), with the greatest distribution being in the 1-5 and 6-10 oocyte collection groups (Figure 5). The main contribution to the first group (low oocyte numbers) came from older women. Very few women had 16-20 oocytes recovered (7.6\%), and an absolute minimum of cases had $>20$ oocytes recovered (4.0\%). Most of the latter cases were attributed to noncompliance with dosing strategy. The number of women with $>15$ oocytes collected at PIVET was 328/2,822 (11.6\%).

Although not the main objective of the study, a direct comparison of the two $\mathrm{rFSH}$ products and consequently the two algorithms for the main outcome measures - efficacy (oocyte and embryo utilization rates and pregnancy and live birth rates) as well as safety (proportion of cases with $>15$ oocytes and OHSS cases) was performed and showed some significant differences (Tables S1-S3). Overall, the data demonstrated that the Puregon group were slightly but significantly older (mean of 0.5 years) with a slightly greater BMI $\left(0.5 \mathrm{~kg} / \mathrm{m}^{2}\right)$ (Table S1). While there were significantly more oocytes and two pronuclei embryos retrieved for the Gonal-f group, there were no significant differences in relation to clinical outcomes such as pregnancy, live births, and miscarriage rates (Table S1). When younger women ( $<40$ years) with lower AMH $(<25 \mathrm{pmol} / \mathrm{L})$ were analyzed, only the numbers of oocytes retrieved and two pronuclei embryos were significantly altered, whereas all other clinical outcomes and oocyte and embryo utilization rates were similar (Table S2). Finally, we investigated the impact of the different $\mathrm{rFSH}$ products on the outcomes in the two main treatment regimens, antagonist and flare agonist cycles (Table S3). No significant difference was observed in the flare cycles, and clinical outcomes (pregnancy, live births, and miscarriage rates) were not altered in the antagonist group. However, in the latter group, the patients receiving Puregon were significantly older and heavier and had a lower oocyte yield and embryo utilization (Table S3).

\section{Discussion}

PIVET is developing unique algorithms to assist clinicians to select appropriate $\mathrm{rFSH}$ dosages with a view to accommodate

Table 4 Clinical pregnancy and live birth outcomes for all women having a TVOA procedure and categorized according to age group

\begin{tabular}{llllll}
\hline & Age group & & & \\
\cline { 2 - 6 } & $<\mathbf{3 0}$ years & $\mathbf{3 0 - 3 4}$ years & $\mathbf{3 5 - 3 9}$ years & $\mathbf{4 0 - 4 4}$ years & $\mathbf{2 4 5}$ years \\
\hline Clinical pregnancy rate per embryo transfer (\%) & 43.4 & 39.9 & 28.6 & 11.7 & 0.0 \\
Live birth rate per embryo transfer (\%) & 36.4 & 33.5 & 22.4 & 7.5 & 0.0 \\
Live birth rate per initiated cycle (\%) & 29 & 27.7 & 18 & 5.5 & 0.0 \\
Freezing rate per initiated cycle (\%) & 64.2 & 64.6 & 51.3 & $\mathbf{2 8 . 4}$ & 9.5 \\
Live birth productivity rate, per initiated cycle (\%) & 57.1 & 51.7 & 33.2 & 8.4 & 0.0 \\
\hline
\end{tabular}

Abbreviation: TVOA, transvaginal oocyte aspiration. 


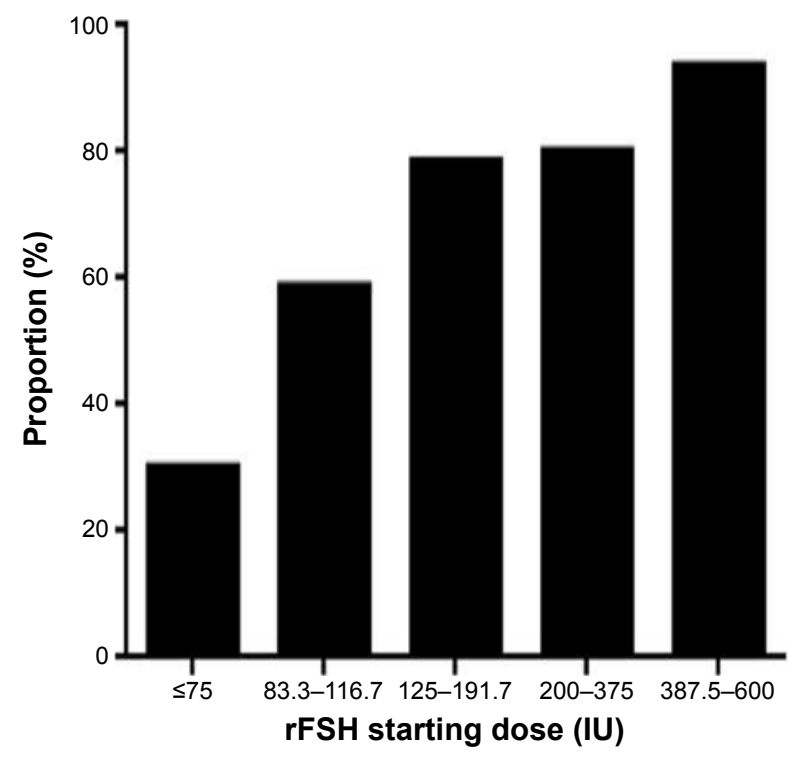

Figure 4 The proportion of patients in each rFSH starting dose category, who responded adequately to the starting dose of $\mathrm{FSH}$ and required no adjustment (elevation) to achieve at least four follicles (for the high AFC groups) and eight to twelve follicles for the remainder.

Note: Where adjustments were made, this was mostly one or occasionally two dose increments.

Abbreviations: AFC, antral follicle count; $\mathrm{rFSH}$, recombinant follicle stimulating hormone.

modern trends of milder stimulation, while maintaining high pregnancy rates, but minimizing or even completely removing the risk of OHSS. Since the first PIVET algorithm was published, ${ }^{5}$ the assisted reproductive technology (ART) world has continued to evolve with several new trends, including the complete removal of OHSS risk by administering antagonist stimulation with GnRH agonist trigger and freeze-all procedures. In addition, there is a strong push, led by Australia and New Zealand, ${ }^{15}$ to commit to an elective single ET policy.

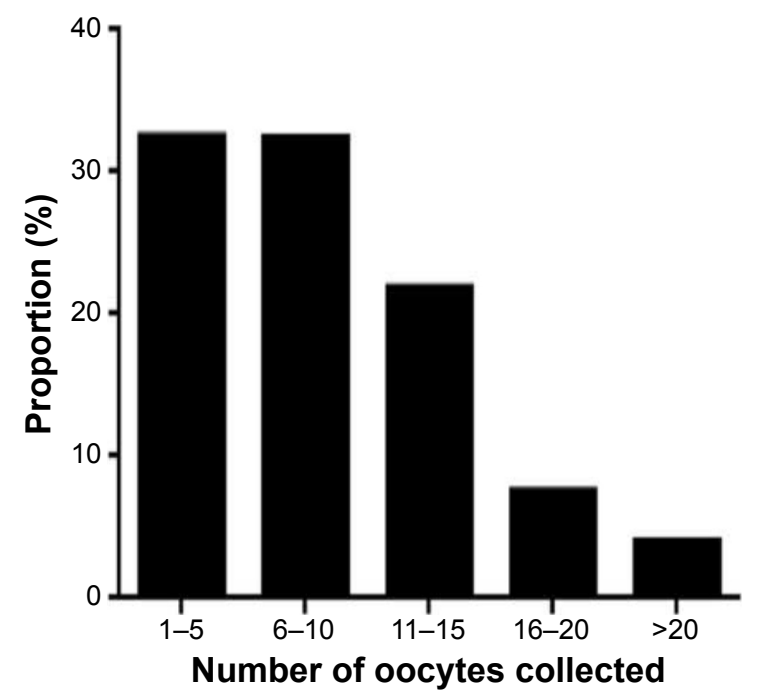

Figure $\mathbf{5}$ The distribution of oocytes recovered, indicating that almost $90 \%$ of women had fewer than 16 oocytes and under $4 \%$ had more than 20 recovered; most resulting from breaches of the described protocols.
Along with that concept, the idea of blastocyst culture with a better embryo selection policy has developed and goes hand-in-glove with the emergent idea of advanced genetic screening of embryos. ${ }^{16,17}$ Consequently, the pregnancy and live birth productivity rates show persisting high levels, in keeping with the oocyte retrieval numbers. ${ }^{6}$ Although it has been shown that 15 oocytes collected per TVOA may be the optimal number for the highest pregnancy and live birth rates from fresh $\mathrm{ETs}^{18,19}$ as well as for pregnancy and live birth productivity rates, ${ }^{6}$ the risks for severe OHSS rise drastically after the collection of twelve or more oocytes. Furthermore, attempts to schedule for 12-15 oocytes retrieval means that many cases will unintentionally result in $\geq 20$ oocytes collected and an exponential rise in OHSS risk. Here, using the PIVET algorithms, only $4 \%$ of cases returned 20 or more oocytes recovered, but these were due to specific protocol violations. The proportion of women with $>15$ oocytes retrieved per egg collection was $11.6 \%$. This was significantly lower than those receiving conventional $\mathrm{rFSH}$ dosages $(112.5-300 \mathrm{IU})$ recently reported ${ }^{20}(38.2 \%)(P<0.0001)$ and demonstrated our milder stimulation.

During the entire study period, OHSS prevention has been the main focus, and other preventive initiatives were also undertaken, including the management of more cycles by an antagonist regimen, which provided the clinician with an opportunity to trigger with a GnRH-agonist and decrease the risk of OHSS. ${ }^{21}$ Furthermore, patients were offered increased monitoring to ensure all patients with OHSS symptoms were detected at the earliest possible stage and that relevant expedient steps could be implemented, such as intravenous fluids to counteract hypovolemia, which were often enough to prevent the complete disorder from developing. The high number of patients on increased monitoring did not necessarily reflect many patients at risk of developing OHSS, but only as a relevant service to detect all cases and expedite early management. In addition, the freeze-all strategy to prevent OHSS in high-risk scenarios ${ }^{22}$ was used throughout the study period. However, despite various preventive strategies, OHSS cases were encountered during the study $(0.3 \%)$, but each and every case was shown retrospectively to be related to noncompliance with the PIVET protocols and regimens as defined in the methodology section. All the cases of severe OHSS were late onset, associated with pregnancy, but also had one of four identifiable noncompliance issues with the advised procedure as defined earlier. We are aware of the controversy over the use of HCG as luteal support, but have data supporting its use to improve the chance of pregnancy ${ }^{23}$ and believe it can be used safely as defined in our policies. 
Its use is clearly safe in women with lower AFC and $\mathrm{AMH}$ levels, for example, categories C, D, and E of the algorithms; perhaps further restrictions need to be considered for the A groups as well as some group B cases. We have now adjusted the protocols to avoid luteal phase HCG in all cases with either AFC rating $\geq 15$ antral follicles or $\mathrm{AMH}$ levels $\geq 15 \mathrm{pmol} / \mathrm{L}$, in addition to the existing restriction of recovery of $\geq 12$ oocytes at TVOA.

High cancellation rates prior to oocyte retrieval can be explained by insufficient follicle development as a result of an inappropriately low rFSH starting dose. In our study, the cancellation rate before TVOA was $6.2 \%$ and included all cancellations after starting rFSH stimulation (ie, initiated cycles). This compared favorably with the overall cancellation rate of 24 of 161 cases $(14.9 \%)$ in the CONSORT algorithm $(P<0.001) .{ }^{4}$ Furthermore, in our study, only 15 of $173(8.7 \%)$ patients were cancelled in the low FSH group ( $\leq 75 \mathrm{IU})$, and this was significantly lower than the $25 \%$ (four of twelve cases) cancellation rate for the CONSORT algorithm, in a potentially good-responder group, requiring low rFSH. ${ }^{4}$ Most cancellations in both series occurred from inadequate responses in the group of younger patients receiving low rFSH dosage.

Moreover, young patients with high $\mathrm{AFC} / \mathrm{AMH}$ often expressed disappointment as they were part of the distribution grouping of one to five oocytes. However, their pregnancy rates were actually high, in keeping with our earlier published data that $<5$ oocytes from younger high-responders generate embryos that have a higher implantation rate than those from higher order oocyte collections. ${ }^{6}$ While those $<30$ years and patients 40-44 years had a mean number of 1.1 and 1.5 embryos transferred, respectively, the overall mean was $1.28 \pm 0.45$, which was significantly lower $(P<0.001)$ than that demonstrated in another study $(1.94 \pm 0.24)$, which was also described as an "optimum oocyte retrieval and transfer strategy." ${ }^{20}$ Furthermore, we noted a trend toward higher embryo utilization rates over oocyte utilization rates in all cases, but both of these rates were increased in older patients. The higher embryo utilization rate reflects the higher number of usable, mature, and fertilizable M-II eggs and is a useful index signifying the proportion of eggs that are actually mature. However, the higher rates in older women may reflect the request by older women to undertake an ET despite lower graded embryos.

Overall both the dosing algorithms, adjusted for $\sim 8.3$ or 12.5 IU increments, have proven effective in meeting the aims of collecting eight to twelve oocytes from the majority of women undergoing ovarian stimulation for IVF procedures. Specifically, the average number of oocytes collected for Gonal-f and Puregon was 9.1 and 8.3, respectively, and 10.1 and 8.0, respectively, for younger women ( $<40$ years) with lower AMH. Although in the overall data set, women receiving Puregon were slightly but significantly older (0.5 years), the reduced oocyte retrieval using Puregon was probably more related to the lower dosage derived from the algorithm, as in the younger group with no significant difference in mean age, the reduced trend in oocyte retrieval was also observed. Additionally, the approximate 8.3 IU subdoses of Puregon are not guaranteed by the manufacturer and could also play a role in these observed differences. Importantly, there was no significant difference in clinical pregnancy, live births, and miscarriage rates between the two rFSH products for all patients collectively or in a subset of younger women $(<40$ years $)$.

In relation to the impact of specific rFSH stimulation protocols, we did not observe any influence of the $\mathrm{rFSH}$ product in flare agonist stimulation, while clinical parameters (pregnancy/livebirths/miscarriage) were also not altered in the antagonist stimulation. However, similar to above, oocyte retrievals were significantly enhanced in the Gonal-f group. The cause of this increase is not known at present but is again probably related to the higher concentrations of Gonal-f used.

Interestingly, the number of cases with $>15$ oocytes was kept at a low of $11.4 \%$ using both of the algorithms, significantly fewer compared to $38.2 \%$ in another recent study ${ }^{20}$ and very few patients exceeded 20 or more oocytes in our study. This is comparable with modern trends of milder stimulation with a view of absolute minimization of the risk of OHSS, with patient safety being of paramount importance. It is our view that such risk can be reduced under $0.01 \%$, but this will require a more determined clinical approach, with stronger adherence to the protocols described here, along with appropriately restricted use of $\mathrm{HCG}$ for triggering in tandem with its careful use in the luteal phase.

Finally, although PIVET has published data showing excellent pregnancy rates from FET cycles, particularly where these are undertaken under hormone replacement therapy control, ${ }^{24}$ we are not supportive of the view that fresh ETs should be avoided. ${ }^{22}$ We believe that the application of the PIVET algorithms provides a rational approach enabling fresh and frozen ETs leading to optimal pregnancy productivity rates, along with the potentially complete avoidance of severe OHSS cases, all being derived from the same TVOA.

\section{Ethical considerations}

General approval for retrospective data analysis was provided from Curtin University (HREC) number RD-25-10 (2015), and 
PIVET Medical Centre is accredited with both the Australian Reproductive Technology Committee (RTAC) and the Reproductive Technology Council (RTC) of Western Australia that functions under statutory regulation. Both the authorities conduct annual accreditation reviews for licencing of ART clinics, with a focus on multiple pregnancies and OHSS rates being of concern if elevated beyond currently adjusted standards.

\section{Acknowledgments}

We wish to acknowledge the relevant contribution of PIVET's previous Scientific Director James D Stanger who helped JLY and PMH develop the first algorithm, which proved a successful idea. The Curtin Health Innovation Research Institute is acknowledged for providing excellent facilities. All participating patients and PIVET staff are acknowledged with thanks. PIVET has self-funded all the facilities, equipment, and manpower for this project.

\section{Author contribution}

JLY and PMH developed the PIVET dosing algorithms. KNK, BA, PMH, JLC and JLY conceived and designed the project. BA, KNK, and PMH extracted the data and undertook the primary analysis. Secondary analysis was performed by KNK, JLC and JLY. BA wrote the first draft, which was revised by KNK, PMH, JLC, and JLY. All authors approve the final version and agree to be accountable for all aspects of the work.

\section{Disclosure}

Although the data presented in this paper concern products from two commercial pharmaceutical companies, we attest that no specific funding was provided for these studies. Along with other companies, MSD and Merck Serono have provided unrelated educational support funding of a limited degree over many years for JLY and PIVET support.

\section{References}

1. Popovic-Todorovic B, Loft A, Bredkjæer HE, Bangsbøll S, Nielsen IK, Andersen AN. A prospective randomized clinical trial comparing an individual dose of recombinant FSH based on predictive factors versus a 'standard' dose of $150 \mathrm{IU} /$ day in 'standard' patients undergoing IVF/ ICSI treatment. Human Reprod. 2003;18(11):2275-2282.

2. Popovic-Todorovic B, Loft A, Lindhard A, Bangsbøll S, Andersson AM, Andersen AN. A prospective study of predictive factors of ovarian response in 'standard' IVF/ICSI patients treated with recombinant FSH. A suggestion for a recombinant FSH dosage normogram. Human Reprod. 2003;18(4):781-787.

3. Howles CM, Saunders H, Alam V, Engrand P. Predictive factors and a corresponding treatment algorithm for controlled ovarian stimulation in patients treated with recombinant human follicle stimulating hormone (Follitropin alfa) during assisted reproduction technology (ART) procedures. An analysis of 1378 patients. Curr Med Res Opin. 2006; 22(5):907-918.
4. Olivennes F, Howles CM, Borini A, et al. Individualizing FSH dose for assisted reproduction using a novel algorithm: the CONSORT study. Reprod Biomed Online. 2009;18(2):195-204.

5. Yovich J, Stanger J, Hinchliffe P. Targeted gonadotrophin stimulation using the PIVET algorithm markedly reduces the risk of OHSS. Reprod Biomed Online. 2012;24(3):281-292.

6. Stanger JD, Yovich JL. Follicle recruitment determines IVF productivity rate via the number of embryos frozen and subsequent transfers. Reprod Biomed Online. 2013;27(3):286-296.

7. Bodri D, Kawachiya S, Brucker MD, et al. Cumulative success rates following mild IVF in unselected infertile patients: a 3-year, singlecentre cohort study. Reprod Biomed Online. 2014;28(5):572-581.

8. Nelson SM, Iliodromiti S, Fleming R, Anderson R, McConnachie A, Messow C-M. Reference range for the antimüllerian hormone Generation II assay: a population study of 10,984 women, with comparison to the established Diagnostics Systems Laboratory nomogram. Fertil Steril. 2014;101(2):523-529.e521.

9. Humaidan P, Ejdrup Bredkjær H, Bungum L, et al. GnRH agonist (buserelin) or hCG for ovulation induction in GnRH antagonist IVF/ICSI cycles: a prospective randomized study. Human Reprod. 2005;20(5):1213-1220.

10. Fisch JD, Keskintepe L, Sher G. Gonadotropin-releasing hormone agonist/ antagonist conversion with estrogen priming in low responders with prior in vitro fertilization failure. Fertil Steril. 2008;89(2):342-347.

11. Nelson SM. Biomarkers of ovarian response: current and future applications. Fertil Steril. 2013;99(4):963-969.

12. Yovich JL, Edirisinghe WR, Cummins JM. Evaluation of luteal support therapy in a randomized controlled study within a GIFT program. Fertil Steril. 1991;55(1):131-139.

13. Yovich JL. Treatments to enhance implantation. In: Chapman M, Grudzinskas JG, Chard T, editors. Implantation: Biological and Clinical Aspects. London: Springer-Verlag; 1989:239-254.

14. Yovich JL, Stanger JD, Keane KN. Cumulative live birth rate: an outmoded term. JFIV Reprod Med Genet. 2016;4:165.

15. Macaldowie A, Wand YA, Chughtai AA, Chambers GM. Assisted Reproductive Technologies in Australia and New Zealand 2012. Sydney: National Perinatal Epidemiology and Statistics Unit, the University of New South Wales; 2014.

16. Fiorentino F, Bono S, Biricik A, et al. Application of next-generation sequencing technology for comprehensive aneuploidy screening of blastocysts in clinical preimplantation genetic screening cycles. Human Reprod. 2014;29(12):2802-2813.

17. Yovich JL, Conceicao J, Hinchliffe P, Keane K. Which blastocysts should be considered for genetic screening? Human Reprod. 2015;30(7): 1743-1744.

18. Ji J, Liu Y, Tong XH, Luo L, Ma J, Chen Z. The optimum number of oocytes in IVF treatment: an analysis of 2455 cycles in China. Human Reprod. 2013;28(10):2728-2734.

19. Sunkara SK, Rittenberg V, Raine-Fenning N, Bhattacharya S, Zamora J, Coomarasamy A. Association between the number of eggs and live birth in IVF treatment: an analysis of 400135 treatment cycles. Human Reprod. 2011;26(7):1768-1774.

20. Chen Y-h, Xu X-h, Wang Q, et al. Optimum oocyte retrieved and transfer strategy in young women with normal ovarian reserve undergoing a long treatment protocol: a retrospective cohort study. $J$ Assist Reprod Genet. 2015;32(10):1459-1467.

21. Humaidan P, Kol S, Papanikolaou E. GnRH agonist for triggering of final oocyte maturation: time for a change of practice? Human Reprod Update. 2011;17(4):510-524.

22. Devroey P, Polyzos NP, Blockeel C. An OHSS-free clinic by segmentation of IVF treatment. Human Reprod. 2011;26(10):2593-2597.

23. Yovich JL, Stanger JD, Yovich JM, Tuvik AI. Hormonal profiles in the follicular phase, luteal phase and first trimester of pregnancies arising from in-vitro fertilization. Br J Obstet Gynaecol. 1985;92(2):374-384.

24. Yovich JL, Conceicao JL, Stanger JD, Hinchliffe PM, Keane KN. Mid-luteal serum progesterone concentrations govern implantation rates for cryopreserved embryo transfers conducted under hormone replacement. Reprod Biomed Online. 2015;31(2):180-191. 


\section{Supplementary materials}

Table SI Clinical outcomes for all patients undergoing rFSH stimulation using either Gonal-f or Puregon

\begin{tabular}{llll}
\hline & Gonal-f ( \pm SD) & Puregon ( \pm SD) & P-value \\
\hline Mean age (years) & $35.9( \pm 5.2)$ & $36.4( \pm 5.4)$ & $0.012^{*}$ \\
Mean BMI (kg/m $\left.\mathrm{m}^{2}\right)$ & $24.4( \pm 4.4)$ & $24.9( \pm 4.5)$ & $0.003^{* *}$ \\
Mean eggs collected & $9.1( \pm 5.7)$ & $8.3( \pm 5.7)$ & $0.0003^{* * *}$ \\
Mean 2PN generated & $5.1( \pm 4.0)$ & $4.7( \pm 3.9)$ & $0.009^{* *}$ \\
Mean oocyte utilization (\%) & $33.3( \pm 23.6)$ & $34.0( \pm 24.3)$ & 0.453 \\
Mean embryo utilization (\%) & $55.3( \pm 32.4)$ & $57.8( \pm 36.7)$ & 0.063 \\
Clinical pregnancy rate per fresh ET (\%) & 28.2 & 28.4 & 0.945 \\
Live birth rate per fresh ET (\%) & 22.8 & 22.4 & 0.838 \\
Miscarriage rate per pregnancy (\%) & 19.0 & 21.2 & 0.551 \\
\hline
\end{tabular}

Notes: No significant differences were observed for pregnancy, birth, or miscarriage rates. However, the populations were slightly but significantly different in terms of mean age, mean $\mathrm{BMI}$, and the number of oocytes and embryos with $2 \mathrm{PN}$ retrieved. $* P<0.05, * * P<0.01$, $* * * P<0.001$.

Abbreviations: 2PN, two pronuclei; BMI, body mass index; $\mathrm{ET}$, embryo transfer; $r F S H$, recombinant follicle stimulating hormone; $\mathrm{SD}$, standard deviation.

Table S2 Clinical outcomes in younger women ( $<40$ years), with $\mathrm{AMH}$ values $<25 \mathrm{pmol} / \mathrm{L}$, receiving $\mathrm{rFSH}$ stimulation using either Gonal-f or Puregon

\begin{tabular}{llll}
\hline & Gonal-f ( \pm SD) & Puregon ( \pm SD) & P-value \\
\hline Mean age (years) & $34.1( \pm 4.0)$ & $34.6( \pm 3.7)$ & 0.115 \\
Mean BMI $\left(\mathrm{kg} / \mathrm{m}^{2}\right)$ & $24.7( \pm 4.5)$ & $25.2( \pm 4.9)$ & 0.196 \\
Mean eggs collected & $10.1( \pm 5.4)$ & $8.0( \pm 5.1)$ & $<0.0001 * * * *$ \\
Mean 2PN generated & $5.7( \pm 4.0)$ & $4.6( \pm 3.7)$ & $0.0007 * * *$ \\
Mean oocyte utilization (\%) & $30.1( \pm 21.5)$ & $31.8( \pm 22.6)$ & 0.357 \\
Mean embryo utilization (\%) & $50.1( \pm 29.8)$ & $53.6( \pm 36.1)$ & 0.204 \\
Clinical pregnancy rate per fresh ET (\%) & 32.8 & 34.9 & 0.693 \\
Live birth rate per fresh ET (\%) & 27.1 & 28.4 & 0.815 \\
Miscarriage rate per pregnancy (\%) & 17.4 & 18.5 & 0.984
\end{tabular}

Note: Only significant differences between number of oocytes collected and number of embryos with $2 \mathrm{PN}$ were observed. $* * * \mathrm{P}<0.00 \mathrm{I}, * * * * \mathrm{P}<0.000 \mathrm{I}$.

Abbreviations: 2PN, two pronuclei; AMH, anti-Müllerian hormone; BMI, body mass index; ET, embryo transfer; rFSH, recombinant follicle stimulating hormone; SD, standard deviation.

Table S3 Clinical outcomes for all patients undergoing rFSH stimulation using either Gonal-f or Puregon and analyzed according to treatment cycle type

\begin{tabular}{|c|c|c|c|c|}
\hline & \multicolumn{2}{|c|}{ Antagonist cycles } & \multicolumn{2}{|c|}{ Flare agonist cycles } \\
\hline & Gonal-f ( \pm SD) & Puregon ( \pm SD) & Gonal-f $( \pm S D)$ & Puregon ( \pm SD) \\
\hline Mean age (years) & $34.3( \pm 4.8)$ & $35.2( \pm 5.4)^{* *}$ & $37.7( \pm 4.5)$ & $37.7( \pm 4.9)^{\mathrm{NS}}$ \\
\hline Mean BMI $\left(\mathrm{kg} / \mathrm{m}^{2}\right)$ & $24.6( \pm 4.6)$ & $25.0( \pm 4.6)^{* * * * *}$ & $24.2( \pm 4.2)$ & $24.8( \pm 4.3)^{\mathrm{NS}}$ \\
\hline Mean eggs collected & $10.4( \pm 5.8)$ & $9.4( \pm 5.6)^{* *}$ & $7.2( \pm 4.6)$ & $7.1( \pm 5.3)^{\mathrm{NS}}$ \\
\hline Mean 2PN generated & $6.0( \pm 4.2)$ & $5.3( \pm 4.0)^{* *}$ & $3.9( \pm 3.2)$ & $3.8( \pm 3.6)^{\mathrm{NS}}$ \\
\hline Mean oocyte utilization (\%) & $30.5( \pm 20.6)$ & $33.1( \pm 21.9)^{\mathrm{NS}}$ & $35.5( \pm 24.6)$ & $34.1( \pm 26.2)^{\mathrm{NS}}$ \\
\hline Mean embryo utilization (\%) & $50.7( \pm 29.6)$ & $56.4( \pm 31.8)^{* *}$ & $60.7( \pm 34.1)$ & $59.7( \pm 43.2)^{\mathrm{NS}}$ \\
\hline Clinical pregnancy rate per fresh ET (\%) & 32.8 & $30.5^{\mathrm{NS}}$ & 22.7 & $25.7^{\text {NS }}$ \\
\hline Live birth rate per fresh ET (\%) & 26.2 & $24.7^{\mathrm{NS}}$ & 19.2 & $19.7^{\mathrm{NS}}$ \\
\hline Miscarriage rate per pregnancy (\%) & 20.0 & $19.0^{\mathrm{NS}}$ & 15.4 & $23.2^{\mathrm{NS}}$ \\
\hline
\end{tabular}

Notes: No significant differences were observed for all parameters using the flare agonist stimulation regimen. For antagonist cycles, the populations were slightly but significantly different in terms of mean age, mean BMI, number of oocytes, and embryos with 2PN retrieved and the embryo utilization rate. However, pregnancy, birth, and miscarriage rates between the two rFSH agents for antagonist cycles were not significantly different. Asterisk indicates significant difference between $r F S H$ drugs in the same cycle type. NS indicates no significant difference between rFSH drugs in the same cycle type.

Abbreviations: 2PN, two pronuclei; BMI, body mass index; ET, embryo transfer; $r F S H$, recombinant follicle stimulating hormone; SD, standard deviation.

Drug Design, Development and Therapy

\section{Publish your work in this journal}

Drug Design, Development and Therapy is an international, peerreviewed open-access journal that spans the spectrum of drug design and development through to clinical applications. Clinical outcomes, patient safety, and programs for the development and effective, safe, and sustained use of medicines are a feature of the journal, which

has also been accepted for indexing on PubMed Central. The manuscript management system is completely online and includes a very quick and fair peer-review system, which is all easy to use. Visit http://www.dovepress.com/testimonials.php to read real quotes from published authors. 